Louisiana State University

LSU Digital Commons

Faculty Publications

Department of Biological Sciences

3-21-2014

\title{
The structure of human 15-lipoxygenase-2 with a substrate mimic
}

Matthew J. Kobe

Louisiana State University

David B. Neau

Argonne National Laboratory

Caitlin E. Mitchell

Louisiana State University

Sue G. Bartlett

Louisiana State University

Marcia E. Newcomer

Louisiana State University

Follow this and additional works at: https://digitalcommons.Isu.edu/biosci_pubs

\section{Recommended Citation}

Kobe, M., Neau, D., Mitchell, C., Bartlett, S., \& Newcomer, M. (2014). The structure of human 15-lipoxygenase-2 with a substrate mimic. Journal of Biological Chemistry, 289 (12), 8562-8569.

https://doi.org/10.1074/jbc.M113.543777

This Article is brought to you for free and open access by the Department of Biological Sciences at LSU Digital Commons. It has been accepted for inclusion in Faculty Publications by an authorized administrator of LSU Digital Commons. For more information, please contact ir@lsu.edu. 


\section{The Structure of Human 15-Lipoxygenase-2 with a Substrate Mimic*}

Received for publication, December 19, 2013, and in revised form, January 31, 2014 Published, JBC Papers in Press, February 4, 2014, DOI 10.1074/jbc.M113.543777

Matthew J. Kobe ${ }^{\ddagger}$, David B. Neau ${ }^{\S}$, Caitlin E. Mitchell ${ }^{\ddagger 1}$, Sue G. Bartlett ${ }^{\ddagger}$, and Marcia E. Newcomer ${ }^{\ddagger 2}$

From the ${ }^{\ddagger}$ Department of Biological Sciences, Louisiana State University, Baton Rouge, Louisiana 70803 and ${ }^{\S}$ Northeastern

Collaborative Access Team, Argonne National Laboratory, Argonne, Illinois 60439

Background: 15-Lipoxygenase-2 is linked to atherosclerotic plaque formation; the homologous enzyme 5-lipoxygenase initiates the synthesis of proinflammatory leukotrienes.

Results: We determined the crystal structure of 15-LOX-2 in the presence of a substrate mimic.

Conclusion: 15-Lipoxygenase-2 and 5-lipoxgenase display active site variations that confer distinct product specificities.

Significance: These differences can be exploited for the design of isoform-specific anti-inflammatories.

Atherosclerosis is associated with chronic inflammation occurring over decades. The enzyme 15-lipoxygenase-2 (15LOX-2) is highly expressed in large atherosclerotic plaques, and its activity has been linked to the progression of macrophages to the lipid-laden foam cells present in atherosclerotic plaques. We report here the crystal structure of human 15-LOX-2 in complex with an inhibitor that appears to bind as a substrate mimic. 15-LOX-2 contains a long loop, composed of hydrophobic amino acids, which projects from the amino-terminal membrane-binding domain. The loop is flanked by two $\mathrm{Ca}^{2+}$-binding sites that confer $\mathrm{Ca}^{2+}$-dependent membrane binding. A comparison of the human 15-LOX-2 and 5-LOX structures reveals similarities at the active sites, as well striking differences that can be exploited for design of isoform-selective inhibitors.

Atherosclerosis is one of several pathologies attributed to an unchecked inflammatory response. A hallmark of the disease is the deposition of macrophage-derived foam cells in plaques that grow to restrict vascular blood flow or rupture. Either event can culminate in one of the various manifestations of cardiovascular disease such as heart attack or stroke. A critical component in the development of atherosclerosis is transformation of macrophages to foam cells, a process involving the uptake of lipids carried by low-density lipoprotein LDL. The presence of oxidized lipids in the LDL particles further exacerbates the process by provoking the release of inflammatory cytokines, attracting still more macrophages to the growing lesion. Mitigation of this inflammatory response can slow the progression of atherosclerosis.

The enzyme 15-lipoxygenase (15-LOX), ${ }^{3}$ which is expressed in macrophages, has been linked to elevated levels of oxidized

* This work was supported in part by National Institutes of Health Grant HL 107887 (to M. E. N.).

The atomic coordinates and structure factors (code 4NRE) have been deposited in the Protein Data Bank (http://wwpdb.org/).

${ }^{1}$ Supported by National Science Foundation Grant MPS 0651345.

${ }^{2}$ To whom correspondence should be addressed: Dept. of Biological Sciences, Louisiana State University, Baton Rouge, LA 70803. Tel.: 225-578738; Fax: 225-578-7258; E-mail: newcomer@|su.edu.

${ }^{3}$ The abbreviations used are: 15-LOX, 15-S-lipoxygenase; AA, arachidonic acid; HPETE, hydroperoxyeicosatetraenoic acid; LOX, lipoxygenase; PC, phosphotidylcholine; PS, phosphotidylserine; HETE, hydroxyeicosatetraenoic acid. lipids through several experimental approaches that include the heterologous expression of human 15-LOX in a mouse model of hyperlipidemia (1) and pharmacological inhibition of 15-LOX activity $(2,3)$. Humans have two 15-LOX (4), designated types 1 (ALOX15A) or 2 (ALOX15B), and the type 1 isoform was the form used in the heterologous expression studies cited above. Mouse knock-out studies with the LOX homologue of 15-LOX-1 support a role for ALOX15A in plaque formation (5). However, it is ALOX15B mRNA that is present in human macrophages $(6,7)$ isolated from atherosclerotic plaques. Furthermore, elevated levels of ALOX15B mRNA are present in carotid lesions derived from symptomatic, rather than asymptomatic, subjects. ALOX15A mRNA is not detected above control levels in either case (6). More recently, Magnusson et al. (8) demonstrated that silencing production of the ALOX15B protein in human macrophages decreased cellular lipid accumulation, the precipitating factor in foam cell formation. Finally, 15S-hydroxyeicosatetraenoic acid (HETE), derived through non-enzymatic reduction of the product of the 15-LOX reaction, has been shown to promote formation of atherosclerotic lesions in a mouse model system (9).

Lipoxygenases (LOX) are a family of iron enzymes that catalyze the peroxidation of polyunsaturated fatty acids to generate oxylipins, which in both animals and plants serve essential roles in signaling (10). The reaction proceeds via abstraction of the hydrogen from the central carbon of a pentadiene and oxidation of the free radical generated two carbons removed from the site of attack. Six distinct LOX have been identified in mammals (4), and the enzymes are named according to their product specificities. Thus, 15-LOX converts the common substrate arachidonic acid (AA) to 15-hydroperoxyeicosatetraenoic acid (HPETE) by attack at C13 of AA, whereas 5-LOX attacks at C7 to transform AA to 5-HPETE (and, subsequently, 5-HPETE to inflammatory leukotriene $\mathrm{A}_{4}$ ). The question of how the AAmetabolizing enzymes are able to discriminate among the three chemically equivalent pentadienes of AA to generate a specific HPETE isomer remains to be clarified.

The various lipid mediators of the cyclooxygenase pathway can have opposing functions. Similarly, the LOX pathways produce either pro- or anti-inflammatory compounds. For example, 5-LOX alone is responsible for the synthesis of the proin- 
flammatory leukotrienes, but anti-inflammatory lipoxins can be synthesized by the combined activities of 15- and 5-LOX or 5- and 12-S-LOX. In addition, 15-LOX also synthesizes the anti-inflammatory neuroprotectin D1 from docosahexaenoic acid (11). These disparate roles for LOX products make development of isoform-specific LOX inhibitors critical in the quest for novel therapeutics.

LOX structures share the common framework established by Boyington et al. (12) with the soybean LOX structure. The animal enzymes, which generally metabolize AA rather than linoleic acid or linolenic acid, represent a pared down version of the fold, as they are $\sim 650$ amino acids in length rather than $\sim 950$ amino acids. However, both the plant and animal enzymes contain an $\mathrm{N}$-terminal membrane binding domain and a C-terminal catalytic domain.

We report here the crystal structure of human 15-LOX-2 in complex with a competitive inhibitor that appears to bind as a substrate mimic. This structure reveals a putative membrane insertion loop and two $\mathrm{Ca}^{2+}$-binding sites also found at the equivalent positions in 5-LOX. Site-directed mutagenesis supports a role for the $\mathrm{Ca}^{2+}$-binding sites in membrane binding, and the unique membrane insertion loop is poised to play a role in tethering the enzyme at the bilayer. Moreover, a comparison of human 15-LOX-2 and 5-LOX structures reveals significant differences in the highly conserved active sites that can be exploited for isoform-specific inhibitor design.

\section{EXPERIMENTAL PROCEDURES}

Plasmid Construction and Protein Expression-15-LOX-2 was co-expressed in Rossetta 2 (DE3) cells in pET Duet-1 with Escherichia coli yjgD protein. The $y j g D$ gene was amplified from E. coli DNA and cloned behind promoter 2 in pET Duet-1 by digesting both vector and insert with NdeI and XhoI. The human 15-LOX-2 cDNA obtained from the ATCC was amplified with primers containing SpeI and HindIII sites and cloned behind promoter 1 by digesting the vector with $\mathrm{XbaI}$ and HindIII. After transformation, Rosetta2 cells (Novagen) were grown as overnight cultures and then diluted (1:100) into 500 $\mathrm{ml}$ volumes of Terrific broth (Alpha Biosciences) in 2-liter Erlenmeyer flasks. After $4 \mathrm{~h}$ at $37^{\circ} \mathrm{C}$ and $220 \mathrm{rpm}$, the speed was reduced to $150 \mathrm{rpm}$, and the cells were incubated an additional $27 \mathrm{~h}$ at $20^{\circ} \mathrm{C}$ for leaky expression of $15-\mathrm{LOX}-2$. Cells were harvested, pelleted, and stored at $-80^{\circ} \mathrm{C}$.

Protein Purification-Cells were lysed in a mixture of Bugbuster (Invitrogen) and Problock 2D protease inhibitor mixture (Gold Biotechnology); both were used according to the manufacturer's instructions. DNase I $(0.2 \mathrm{mg} / \mathrm{ml})$ (Sigma) was added, and the suspension was processed in a French pressure cell. Insoluble material was removed by centrifugation at 17,500 rpm for $30 \mathrm{~min}$, and imidazole buffer was added to the supernatant to a final concentration of $20 \mathrm{~mm}$. The sample was syringe-filtered $(0.45 \mu \mathrm{M}$ pore size $)$ and applied to a $\mathrm{Co}^{2+}{ }^{2}$-nitroloacetic acid column (Pierce) equilibrated with buffer A (20 $\mathrm{mm}$ Tris- $\mathrm{HCl}, \mathrm{pH} 8.0,20 \mathrm{~mm}$ imidazole, $500 \mathrm{~mm} \mathrm{NaCl}$ ) and configured on an Akta FPLC (GE Healthcare). Protein was eluted with a $0-100 \%$ gradient of buffer B $(20 \mathrm{~mm}$ Tris- $\mathrm{HCl}, \mathrm{pH}$ 8.0, $200 \mathrm{~mm}$ imidazole, $500 \mathrm{~mm} \mathrm{NaCl}$ ). The major peak eluted from the column at $\sim 30 \% \mathrm{~B}$. Peak fractions were pooled and concentrated with a 35-kDa Amicon ultra centrifugal filtration unit (Sigma) and subsequently applied to Superdex 200 (GE Healthcare) for gel filtration. SDS-PAGE, enzyme assays, and product analysis confirmed that the major peak which eluted at $86 \mathrm{ml}$ corresponded to 15-LOX-2. Protein was concentrated to $\sim 10 \mathrm{mg} / \mathrm{ml}$, flash-frozen dropwise in liquid $\mathrm{N}_{2}$, and stored at $-80^{\circ} \mathrm{C}$ until use.

Crystallization-15-LOX-2 was screened for crystallization conditions with crystal screens from Hampton and a Gryphon liquid dispenser (ARI). Conditions yielding crystalline-like material were further screened varying $\mathrm{pH}$, buffer, cryo-protectant, and detergent. After extensive additive screening, the detergent octyltetraethylene glycol ether (C8E4, Anatrace) proved essential for crystallization. The conditions that yielded diffraction quality rhombohedral crystals were $1 \mathrm{M} \mathrm{NH}_{4} \mathrm{SO}_{4}, 0.1$ м Bis-Tris, pH 5.5, 5\% glycerol, and 24 mм C8E4. Crystals were grown in hanging drops.

Diffraction Data Collection-15-LOX-2 crystals were looped from the mother liquor and placed directly in the $100 \mathrm{~K} \mathrm{~N}_{2}$ stream of an Oxford CryoJet or plunged into liquid $\mathrm{N}_{2}$ for shipping. Two x-ray data sets were collected at separate beamlines. The first data set was collected to a resolution of $2.85 \AA$ at the Gulf Coast Protein Crystallography Consortium beamline at the Center for Advanced Microstructures and Devices (Baton Rouge, LA). The final data set was collected to $2.65 \AA$ at the 24ID-E beamline of the Northeastern Collaborative Access Team at the Advanced Photon Source (Argonne National Laboratory). This last data set was obtained from a crystal soaked for $30 \mathrm{~min}$ in mother liquor supplemented with $40 \mathrm{mM} \mathrm{CaCl}_{2}$ prior to flash-cooling in liquid $\mathrm{N}_{2}$. HKL2000 (13) was used to process data collected at the Gulf Coast Protein Crystallography Consortium beamline. XDS, pointless, and scala were used via the RAPD processing suite of the Northeastern Collaborative Access team to process the data collected by the Northeastern Collaborative Access Team.

The Phenix program suite (14) was used to perform molecular replacement (with PHASER) and the model refinement that followed. Model building was performed in Coot (15). The structure of Stable-5- LOX (Protein Data Bank 3O8Y) served as the search model for the $2.85 \AA$ data set. The model constructed with this data set was subsequently used as the search model for the $2.65 \AA$ data from a $\mathrm{CaCl}_{2}$ soaked crystal. Refinement was performed with Phenix.refine.

Enzyme Assays-Enzyme activity was monitored in an Applied Photophysics Model SX.18MV stopped-flow spectrometer at $22^{\circ} \mathrm{C}$. Product (15-hydroperoxyeicosatetraenoic acid) formation was monitored at $235 \mathrm{~nm}$ with a cell path length of $0.2 \mathrm{~cm}$. The enzyme concentration was $60 \mathrm{~nm}$ in $20 \mathrm{~mm}$ Tris- $\mathrm{HCl}$ (pH 7.5), $150 \mathrm{~mm} \mathrm{NaCl}$. Assays were performed with arachidonic acid (Cayman Chemical) concentrations ranging from 1 to $40 \mu \mathrm{M}$. The reported concentrations of reagents are those in the observation cell. Each kinetic trace was the average of at least three individual measurements. $K_{m}$ and $V_{\max }$ value were determined by non-linear regression analysis of a plot of velocity versus substrate concentration to Equation 1. $V_{\max }$ is the maximal velocity, $S$ is the substrate concentration, and $K_{m}$ is the Michaelis constant. 


$$
\nu=\frac{V_{\max } \times[S]}{K_{m}+[S]}
$$

Kinetic assays in the presence of C8E4 were performed to determine the mode of inhibition. In these assays, the concentration of AA was varied between 1-40 $\mu \mathrm{M}$ in the presence of varying concentrations of C8E4 detergent. The enzyme concentration was $100 \mathrm{~nm}$. Nonlinear regression was used to analyze the stopped-flow data (16) obtained as described above. Each data point is the average of at least three kinetic traces. The data were fit to Equation 2, where $v$ is the initial velocity, $V_{\max }$ is the maximal velocity, $S$ is the substrate concentration, $K_{m}$ is the Michaelis constant, $\left[I\right.$ is the inhibitor concentration, and $K_{i}$ is the inhibition constant.

$$
\nu=\frac{V_{\max } \times[S]}{K_{m}\left(1+\frac{[I]}{K_{i}}\right)+[S]}
$$

Nanodisc Preparation-Nanodiscs were prepared with MSP1E3D1 and phospholipids as described $(17,18)$ with minor modifications. Briefly, plasmid pMSP1E3D1 (Addgene) was expressed in Rosetta 2 cells in Terrific broth $\left(37^{\circ} \mathrm{C}\right)$ in the presence of $30 \mu \mathrm{g} / \mathrm{ml}$ kanamycin. Expression was induced with 0.5 mM isopropyl 1-thio- $\beta$-D-galactopyranoside at $A_{600}$ of 1.4 , and the cells were allowed to grow for $3.5 \mathrm{~h}$. The temperature was lowered to $28^{\circ} \mathrm{C} 1 \mathrm{~h}$ post induction. Cells were pelleted and lysed by sonication in $50 \mathrm{~mm}$ sodium phosphate (7.4), $1 \mathrm{~mm}$ PMSF, 1\% Triton X-100. Cell debris was pelleted, and the lysate was applied to a nickel chelate affinity column. The column was washed with five volumes of 1 ) buffer $\mathrm{C}(40 \mathrm{~mm}$ Tris ( $\mathrm{pH}$ 8.0), $300 \mathrm{~mm} \mathrm{NaCl}$ ); 2) plus 1\% Triton X-100; 3) plus $50 \mathrm{~mm}$ cholate; 4) buffer $\mathrm{C}$, and 5) plus $50 \mathrm{~mm}$ imidazole. Protein was eluted with buffer $\mathrm{C}$ containing $300 \mathrm{~mm}$ imidazole. MSP1E3D1-containing fractions were pooled, concentrated to at least $5 \mathrm{mg} / \mathrm{ml}$, dialyzed into nanodisc buffer (20 mM Tris- $\mathrm{HCl}, \mathrm{pH} 7.4,150 \mathrm{~mm}$ $\mathrm{NaCl}, 0.5 \mathrm{~mm}$ EDTA), and lyophilized.

Nanodiscs were prepared from rehydrated MSP aliquots, and phospholipids were resuspended in nanodisc buffer containing $100 \mathrm{~mm}$ cholate. Mixtures of phospholipids (3:1 porcine brain PC, porcine brain PS, Avanti Polar Lipids) and MSP (160:1) in nanodisc buffer with $25 \mathrm{~mm}$ cholate were incubated for $15 \mathrm{~min}$ at room temperature. Cholate removal to induce nanodisc formation was accomplished with the addition of Biobeads $(0.8 \mathrm{~g} / \mathrm{ml}$, Bio-Rad) and incubation at room temperature for $2 \mathrm{~h}$. Biobeads were removed by centrifugation. Nanodisc formation and nanodisc binding by 15-LOX-2 were monitored by size exclusion chromatography on a Superose- 12 column (GE Healthcare).

\section{RESULTS}

A Unique $\beta$-Hairpin Structure Projects from the Membranebinding Domain-The crystal structure of human 15-LOX-2 was determined at $2.65 \AA$ resolution $\left(R_{\text {work }} / R_{\text {free }}=16.3 / 20.3\right.$; Table 1). As expected, 15-LOX-2 displays the typical LOX fold (Fig. 1, $A$ and $B$ ): an amino-terminal $\beta$-barrel domain, which is known as a PLAT domain (polycystin-1-lipoxygenase $\alpha$-toxin), and a significantly larger $\alpha$-helical catalytic domain. The cata-
TABLE 1

\begin{tabular}{|c|c|c|}
\hline & $\begin{array}{c}\text { 15-LOX-2 (Protein } \\
\text { Data Bank code 4NRE) } \\
\left(40 \mathrm{mM} \mathrm{CaCl}_{2} \text { soak }\right)\end{array}$ & 15-LOX-2 \\
\hline \multicolumn{3}{|l|}{ Data collection } \\
\hline Space group & R32 & R32 \\
\hline Cell dimensions & $\begin{array}{c}a=155.79, b=155.79, \text { and } \\
c=263.21 \AA ⿻ \alpha=90, \\
\beta=90, \text { and } \gamma=120^{\circ}\end{array}$ & $\begin{array}{c}a=154.6, b=154.6, \\
c=262.78 \AA ; \alpha=90, \\
\beta=90, \gamma=120^{\circ}\end{array}$ \\
\hline Wavelength & 0.97923 & 1.38079 \\
\hline Resolution $(\AA)$ & $38.95-2.63(2.72-2.63)$ & $50.0-2.72(2.83-2.72)$ \\
\hline$R_{\text {merge }}$ & $0.048(0.39)$ & $0.20(0.55)$ \\
\hline$I / \sigma I$ & $14.2(2.0)$ & $11.95(1.9)$ \\
\hline Completeness (\%) & $94.07(68.7)$ & $99.9(100)$ \\
\hline Redundancy & $2.6(2.1)$ & $4.9(4.7)$ \\
\hline \multicolumn{3}{|l|}{ Refinement } \\
\hline Resolution $(\AA)$ & $38.95-2.63(2.72-2.63)$ & \\
\hline No. reflections & $34,565(2409)$ & \\
\hline$R_{\text {work }} / R_{\text {free }}$ & $16.27 / 20.33(26.66 / 29.94)$ & \\
\hline No. atoms & 5882 & \\
\hline Protein & 5385 & \\
\hline Water & 368 & \\
\hline Iron & 1 & \\
\hline Calcium & 2 & \\
\hline Ligand (C8E4) & 12 & \\
\hline \multicolumn{3}{|l|}{$B$-factors $\left(\AA^{2}\right)$} \\
\hline Protein & 61.6 & \\
\hline C8E4 & 56.6 & \\
\hline Calcium & 75.6 & \\
\hline Iron & 47.4 & \\
\hline Solvent & 57.2 & \\
\hline Wilson B & 50.5 & \\
\hline \multicolumn{3}{|l|}{ r.m.s.d. ${ }^{a}$} \\
\hline Bond lengths $(\AA)$ & 0.005 & \\
\hline Bond angles & $0.9^{\circ}$ & \\
\hline \multicolumn{3}{|l|}{ Ramachandran } \\
\hline Outliers (\%) & 0.0 & \\
\hline Favored (\%) & 97 & \\
\hline
\end{tabular}

Diffraction data and structure refinement

${ }^{a}$ r.m.s.d., root mean square deviation.
A

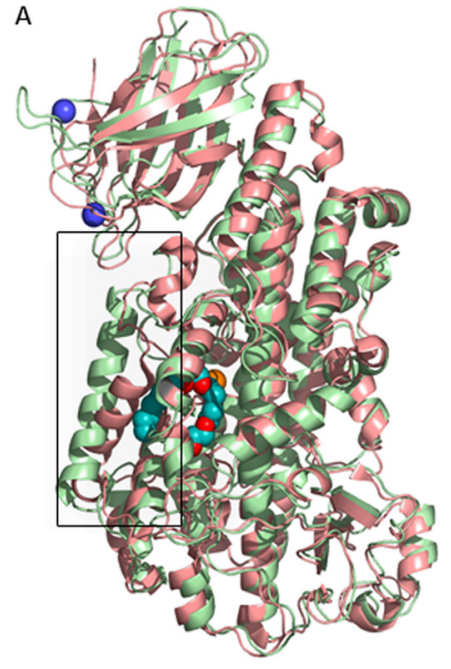

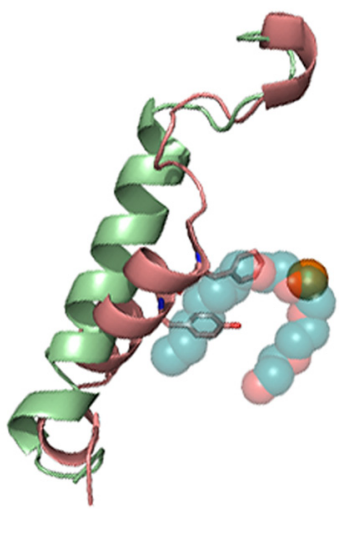

FIGURE 1. Overlay of 15-LOX-2 and stable 5-LOX. $A$, schematic renderings of 15-LOX-2 (green) and stable 5-LOX (pink). $\mathrm{Ca}^{2+}$ is shown as blue spheres, and the catalytic iron is in rust. $B$, detail of the difference in positioning of helix $\alpha 2$. Side chains from the 5-LOX segment occupy the shallower half of the 15-LOX-2 inhibitor binding site.

lytic iron is positioned by the side chains of three invariant histidines (at positions 373, 378, and 553) and the main chain carboxyl of the $\mathrm{C}$ terminus $\left(\mathrm{Ile}^{676}\right)$. Human 15-LOX-2 shares $42 \%$ sequence identity with human 5 -LOX and $38 \%$ or less than that with any other LOX for which a structure has been reported (19-24). 
The Structure of 15-Lipoxygenase-2

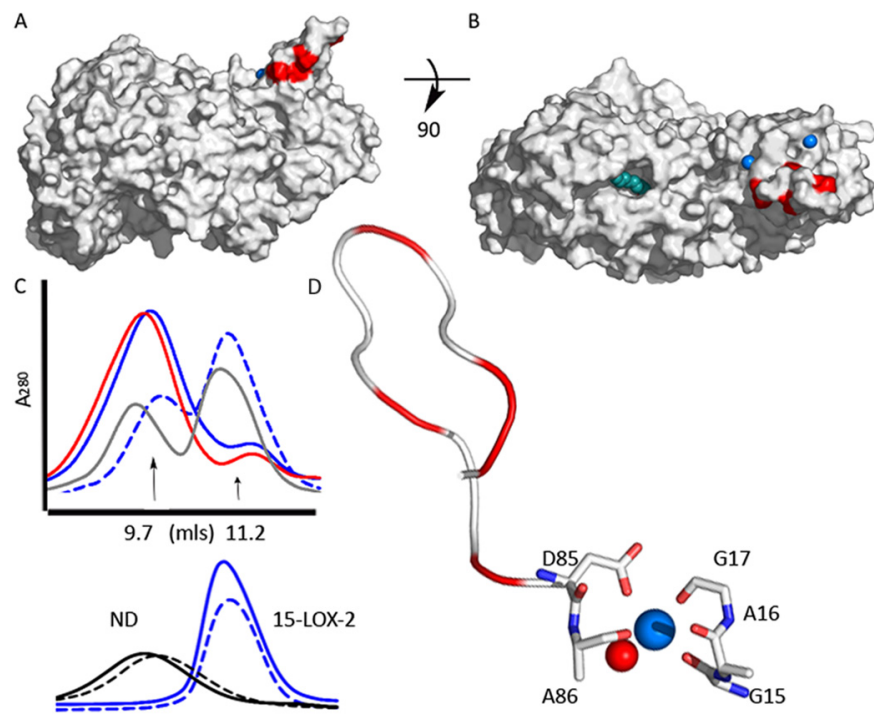

FIGURE 2. 15-LOX-2 and membrane binding. $A$, surface rendering of 15-LOX-2. The putative membrane insertion loop projects from the aminoterminal PLAT domain; prolines present in the loop are colored in red. $B$, rotation $90^{\circ}$ about the horizontal, looking into the active site (C8E4, teal). The $\mathrm{Ca}^{2+}$-binding sites (blue spheres) are on the same face of the molecule as the active site cavity, which is open to solvent. $C$, upper panel: in the presence of $\mathrm{Ca}^{2+}, 15-\mathrm{LOX}-2$ co-elutes with nanodiscs. Dashed line, $0.5 \mathrm{~mm}$ EDTA; solid lines, plus $2 \mathrm{~mm} \mathrm{CaCl}_{2}$. Blue, wild-type; red, D39A/E44A; gray, D39A/E44A/D85A. Lower panel: for reference, individual elution profiles for nanodiscs (ND; black) and 15-LOX-2 (blue); dashed line, $0.5 \mathrm{~mm}$ EDTA; solid line, plus $2.0 \mathrm{~mm} \mathrm{Ca}^{2+}$. D, detail of the $\mathrm{Ca}^{2+}$-binding site that anchors the insertion loop. Three main chain carbonyls, $\mathrm{Asp}^{85}$, and water (red spheres) coordinate the $\mathrm{Ca}^{2+}$ (blue spheres).

LOXs are cylindrical in overall shape $(\sim 100 \AA$ in length, $\sim 45$ $\AA$ in width), and the PLAT domain is roughly one-third of the cylinder. In 15-LOX-2, the PLAT domain contains two apparent $\mathrm{Ca}^{2+}$-binding sites, the first of which $(\mathrm{Ca}-\mathrm{l})$ is located in the turn at amino acids $39-44$. The $\mathrm{Ca}^{2+}$ in Ca- 1 is coordinated by $\mathrm{Asp}^{39}$ and $\mathrm{Glu}^{44}$ and the main chain carbonyls of $\mathrm{Asn}^{40}$ and $\mathrm{Gly}^{42}$ and a water molecule. The second site (Ca-2) appears to stabilize an $\sim 12$-amino acid loop at $\operatorname{Pro}^{74}-\mathrm{Ala}^{86}$ that connects strands from the two sides of the stacked $\beta$-sheets of the barrel. This proline-rich hydrophobic loop projects $20 \AA$ from the membrane-binding domain as a hairpin-like structure. The $\mathrm{Ca}-2$ site, which is coordinated by $\mathrm{Asp}^{85}$, the main chain carbonyls of $\mathrm{Ala}^{86}, \mathrm{Gly}^{15}, \mathrm{Gly}^{17}$, and a water molecule, appears to anchor the C-terminal region of the hairpin loop. The loop is restrained by a crystal-packing contact in the crystal structure, as it is nestled at the active site entrance of a neighboring molecule. Although this interaction may constrain the loop in an extended fashion, the prolines at its base and within the sequence also provide rigidity and contribute to its projection from the body of the protein. The projection of the loop from the body of the protein is visible in the surface rendering in Fig. 2, $A$ and $B$.

Phospholipase $\mathrm{A}_{2}$ and human 5-LOX contain highly conserved calcium binding sites in their PLAT domains $(13,19,20)$. Although sequence homology is low, the 15-LOX-2 Ca-1 and $\mathrm{Ca}-2$ binding sites are in equivalent positions with those in 5 -LOX, which displays $\mathrm{Ca}^{2+}$-dependent membrane binding $(25,26)$. The $\mathrm{Ca}^{2+}$-binding sites are positioned along one edge of the PLAT domain, on the same face of the elongated molecule that harbors the entrance to the catalytic site. These obser-
TABLE 2

15-LOX-2 kinetic parameters

\begin{tabular}{lcc}
\hline \multicolumn{1}{c}{ Enzyme } & $\boldsymbol{K}_{\boldsymbol{m}}$ & $\boldsymbol{k}_{\text {cat }}$ \\
\hline & $\mu M$ & $s^{-1}$ \\
Wild type & $1.9 \pm 0.37$ & $0.6 \pm 0.02$ \\
D39A/E44A & $1.7 \pm 0.16$ & $0.5 \pm 0.01$ \\
D39A: D3 & $0.76 \pm 0.06$ & $0.6 \pm 0.01$ \\
D39A/E44A/D85A & 0.5 & \\
\hline
\end{tabular}

vations led us to test whether $\mathrm{Asp}^{39}, \mathrm{Glu}^{44}$, and $\mathrm{Asp}^{85}$ confer $\mathrm{Ca}^{2+}$-dependent membrane binding of 15-LOX-2 (27).

$\mathrm{Ca}^{2+}$-dependent Membrane Binding- $\mathrm{Ca}^{2+}$-dependent membrane binding was monitored using nanodiscs as membrane mimics. Nanodiscs with a diameter of $\sim 110 \AA$ were prepared with membrane scaffold protein E3. In the absence and presence of $\mathrm{Ca}^{2+}$ nanodiscs and 15-LOX-2 elute at $\sim 9.7$ and $\sim 11.2 \mathrm{ml}$, respectively, on a Superose 12 column (Fig. 2 C). In the absence of $\mathrm{Ca}^{2+}$, when co-incubated mixtures are applied to the sizing column, nanodiscs, and $15-\mathrm{LOX}-2$ retain their individual elution volumes. In contrast, with the addition of 2 $\mathrm{mM} \mathrm{CaCl}_{2}$ to the elution buffer (which is $0.5 \mathrm{~mm}$ EDTA), the LOX peak shifts to indicate co-elution with nanodiscs. This same shift was observed in a mutant that lacks Ca-1 (D39A/ E44A). In contrast, a mutant lacking both calcium sites (D39A/ E44A/D85A) eluted at a volume consistent with free enzyme (Fig. 2C). Mutations of $\mathrm{Ca}^{2+}$-binding amino acids does not significantly affect enzyme activity (Table 2). All enzymes display the same $k_{\text {cat }}$ an indication that the catalytic machinery remains intact. A detail of the $\mathrm{Ca}-2$ site, which anchors the extended loop, is depicted in Fig. $2 D$.

The Crystal Structure of 15-LOX-2 Reveals a Novel Inhibitor Binding in a Substrate-like Conformation-The LOX catalytic domain is a bundle of $\sim 20$ helices. Although LOXs share a common fold and the conformations and placement of the majority of the helices are conserved, two distinct configurations have been described for the region that constitutes the second helix $(\alpha 2)$ in this domain (amino acids 178-199 in 15-LOX-2). In 15-LOX-1 (20), 8R-LOX (23) and 12-LOX (21), this region is a single $\alpha$-helix that extends nearly the length of the catalytic domain and rims the active site cavity. In contrast, the same segment in the 5-LOX (19) and 11R-LOX (22) structures is a meandering, broken helix that seals off the cavity. The structure of human 15-LOX-2 displays the first orientation: a single $\alpha$-helix that helps define the periphery of the active site (see Fig. 1). The opposite rim of the active site is formed by a structurally conserved "arched" helix (amino acids 410 - 430 in 15-LOX-2).

In the first experimental map calculated with data to $2.8 \AA$ resolution, a continuous electron density that could not be attributed to polypeptide was positioned above the catalytic iron. The polyoxyethylene detergent C8E4, which was required for diffraction quality crystals, was the most likely candidate for this density. A molecule of C8E4 was fit into the density such that the polyoxyethylene tail was innermost and the hydrocarbon end made its way out of the active site through the entrance portal defined by $\alpha 2$ and the arched helix (Fig. $3 A$ ). The higher sigma value of the innermost electron density most likely represents the O-rich end of the detergent, but it is not possible to definitively determine the orientation of $\mathrm{C} 8 \mathrm{E} 4$ in the active site. 
A

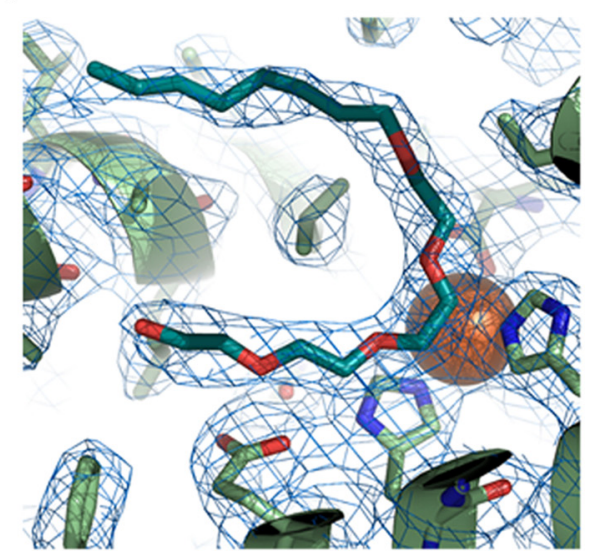

B

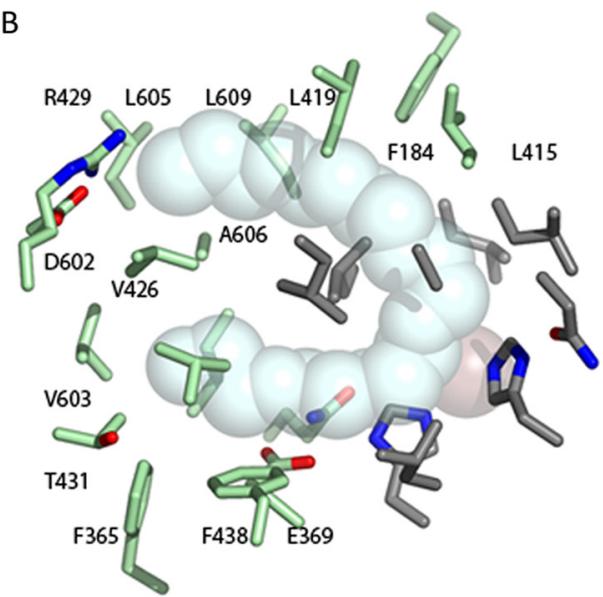

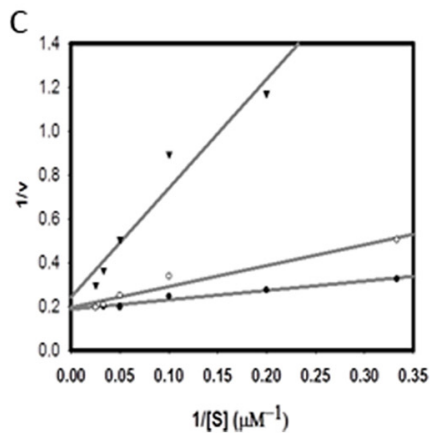
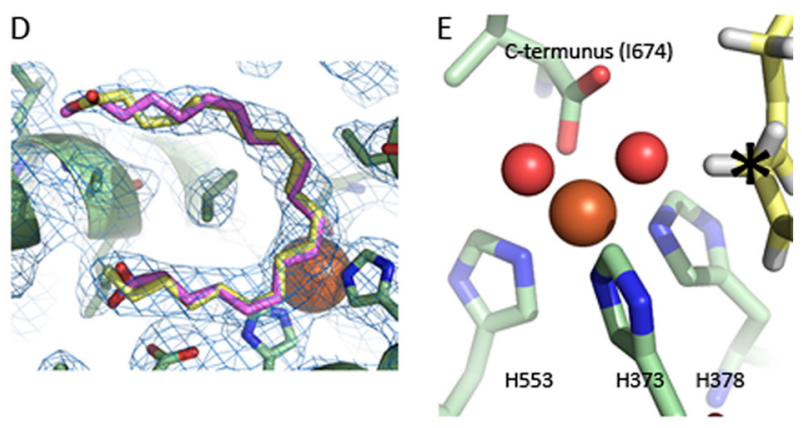

FIGURE 3. The structure was determined in the presence of a competitive inhibitor. $A$, omit map (calculated without C8E4) electron density ( $2 F_{o}-F_{c}, 1 \sigma$ ) positioned above the active site iron is consistent with the detergent C8E4. $B$, the amino acids that line the active site cavity. Shown is atomic coloring (green, carbon; red, oxygen; blue, nitrogen), with invariant/highly-conserved amino acids in gray. $C$, non-linear regression analysis of enzyme activity measured at varying inhibitor and substrate concentrations. (, $0 \mathrm{~mm} ; \mathrm{O}, 0.1 \mathrm{~mm} ; \mathbf{\nabla}, 1.0 \mathrm{~mm} C 8 \mathrm{E} 4$; AA from 1 to $40 \mu \mathrm{m}$ ). The points represent the observed velocities, and the lines represent the best fit of the data to Equation 2. D, AA (purple and yellow stick renderings; red, oxygen) can be modeled into the C8E4 density in two inversely related orientations. E, detail of the placement of the yellow AA in D. The iron (rust) coordination sphere is filled by His-373, His-378, His-553, the carboxyl terminus, and two water molecules $(r e d)$. The hydrogen of the pentadiene is poised for abstraction. The asterisk marks C13. The inverse fit of AA C7 sits at this position.

(The Protein Data Bank file contains a second detergent modeled on the surface of the protein. Based on the paucity of contacts it makes with the protein and the high concentration of detergent in the crystallization buffer, this is likely a nonspecific protein-detergent interaction.)

The active site C8E4 sits in the cavity in a U-shaped conformation and is surrounded primarily by the side chains of hydrophobic amino acids (Fig. 3B). Proximal to the base of the "U" sits the catalytic iron, held in place by invariant histidines 373,378 , and 553. Given the shape of the cavity, we asked whether C8E4 might mimic the conformation of the natural substrate AA and serve as a competitive inhibitor. To test this hypothesis, enzyme activity was assayed by monitoring the increase in absorbance $(235 \mathrm{~nm})$ of HPETE at varying C8E4 concentrations. The data were fit to Equation 2. Non-linear regression analysis by the method of Cleland (16) indicates the $K_{i}$ for C8E4 is $92( \pm 16) \mu \mathrm{M}$ (Fig. 3C), and the inhibition is competitive. In contrast, a satisfactory fit to the equation for non-competitive inhibition was not obtainable.

Modeling of AA in the Active Site-Given that C8E4 competitively inhibits 15 -LOX-2, we asked whether the substrate might fit into the volume occupied by the detergent. Two orientations are possible: one with the carboxyl ("head") innermost and the other the hydrocarbon ("tail") end innermost. We refined both orientations in the C8E4 electron density with the refine ligand option in PHENIX. As can be seen from Fig. 3D, both fit into the C8E4 density, suggesting that the detergent bound structure is similar to that of the substrate-bound structure.

Mechanistic studies have demonstrated that abstraction of the hydrogen from the central carbon of the pentadiene and oxidation occur on opposite faces of the AA (see Ref. 28). LOX structures provide a framework to envision why this is necessary, as the same amino acids that position the catalytic iron shield the pentadiene from molecular oxygen (Fig. 3). Crystal structures of LOX reveal the enzyme resting state in which in $\mathrm{Fe}^{2+}$ occupies the metal binding site. The enzyme must be activated to $\mathrm{Fe}^{3+}$ to proceed with catalysis as it is $\mathrm{Fe}^{3+} \mathrm{OH}^{-}$that abstracts the $\mathrm{H}$ from the pentadiene (see Ref. 29). Note that in Fig. $3 E$, the $\mathrm{Fe}^{2+}$ coordination is completed with two waters. One of these waters is poised to strike a substrate $\mathrm{H}$ on the central carbon of the positioned pentadiene (Fig. 3E). In the "tail first" fit, this is $\mathrm{C} 13$, whereas in the head first orientation, it is $\mathrm{C} 7$.

Fig 4, $A$ and $B$, provides a view of the relative placements of the substrate binding site, the $\mathrm{O}_{2}$ channel (30), and the catalytic iron. Although AA can be modeled in two orientations that are "inversely" related, only one orientation is consistent with the product specificity of 15-LOX-2. A schematic provided Fig. 4C summarizes this information. Note the "inverse" orientations 
A

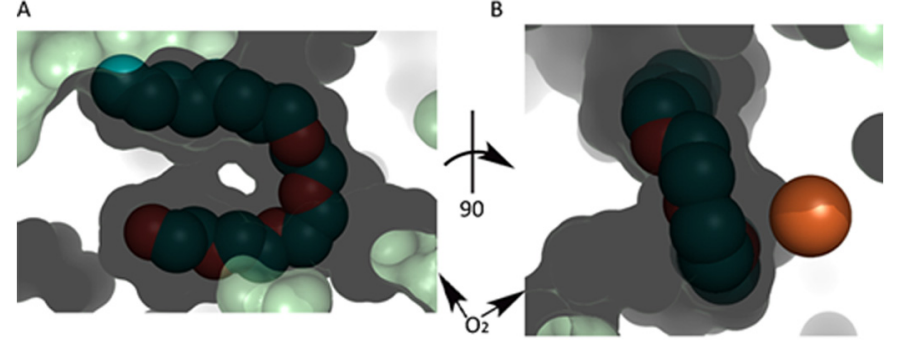

C

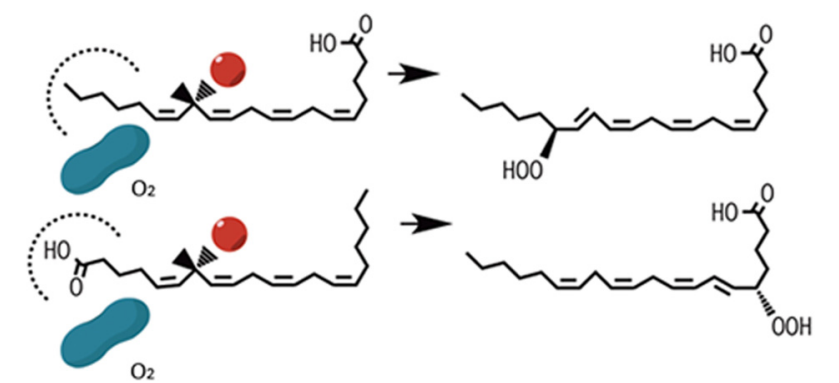

FIGURE 4. Mechanistic details are consistent with a unique orientation of AA in the active site. $A$, the contour of the active site with bound C8E4 is complementary to AA in shape, and the catalytic iron and apparent oxygen channel lie on opposite sides of the tunnel. This relationship between $\mathrm{O}_{2}$ channel and catalytic iron is shown clearly in $B$. $C$, schematic of the two fits (Fig. 3D) of AA. The substrate can be modeled it with its tail (top) or head deepest in the cavity (bottom). The depth of the pocket and the orientation of the substrate determine which pentadiene is positioned for attack. In the top orientation, the pentadiene at C 13 is positioned for attack; in the lower, C7 is positioned proximal to the red sphere (iron) behind the plane. The blue peanut represents the $\mathrm{O}_{2}$ channel slightly deeper in the active site but above the plane. A single product is produced by each orientation: with the tail end innermost AA is transformed to 15-S-HPETE, whereas the inverse orientation results in 5-S-HPETE.

of AA on the left side of the figure. Both AAs are positioned such that the iron (red sphere) and $\mathrm{O}_{2}$ channel (blue peanut) are on opposite sides, but in the upper one, $\mathrm{C} 13$ is positioned for attack and in the lower C7. (It is not possible for either orientation to slide deeper in the pocket to produce another regiochemistry.) To produce 15-HPETE, hydrogen abstraction must occur at C13, which is only possible with tail first entry. Note that abstraction of the "back" hydrogen dictates oxygenation on the "front." The top product is 15-S-HPETE, and the lower product is 5-S-HEPTE. So the first orientation has both the correct regio- and stereo-chemistries because 15-LOX-2 generates 15-S-HPETE exclusively. Moreover, 15-LOX-2 can process phospholipid-esterified AA (31). This observation is not possible if the carboxyl end were innermost in the site because there is no room for the phospholipid head group.

In conclusion, the detergent defines a cavity that can accommodate AA with no further conformational changes. Moreover, the substrate pentadiene centered at $\mathrm{C} 13$ is positioned for attack. An $\mathrm{O}_{2}$ channel lies slightly deeper in the cavity to allow $\mathrm{O}_{2}$ access to $\mathrm{C} 15$. The stereochemistry of the product for this model is what is observed in vivo. The structure is entirely consistent with tail-first entry, and inconsistent with head-first entry of substrate.

\section{DISCUSSION}

Several lines of evidence indicate a link between 15-LOX activity and atherosclerosis. Macrophage uptake of oxidized low-density lipoprotein promotes their progression to lipid-loaded foam cells that contribute to atherosclerotic plaques, and oxidized low- density lipoprotein levels correlate with 15-LOX activity. A model for how an intracellular enzyme such as 15-LOX can contribute to the oxidation of extracellular LDL has been proposed by Hutchins and Murphy (32). The investigators identified 15-LOX products of the cholesterol esters of deuterated AA and linoleic acid in macrophages incubated with lipid vesicles of cholesterol ester-esterified arachidonic and linoleic acids. Their results led them to suggest that the hydroperoxy acid products generated by cytosolic 15-LOX enter into extracellular pools of cholesterol esters and phospholipids through both the continuous remodeling of cholesterol esters and phospholipids and the constant trafficking of lipids among cellular compartments.

Two 15 -lipoxygenases, with $<40 \%$ sequence identity and distinct patterns of expression and product profiles, have been identified in humans $(27,33)$. Human 15-LOX-1 generates 15-HPETE, and to a lesser extent 12-HPETE using AA as a substrate, whereas the product of 15-LOX-2 with AA is exclusively 15-HPETE (33). Recent data implicate the 15-LOX-2 isoform in the pathogenesis of atherosclerosis because mRNA and protein levels of this LOX have been shown to be high in human carotid plaques (6). In addition, 15-LOX-2 is expressed in macrophages $(7,34,35)$, and expression is regulated by hypoxia-inducible factor- $1 \alpha$ (35). Moreover, knockdown of 15-LOX-2 expression in human primary macrophages and in mice (in this case, the target was 8-LOX, the murine homologue of 15-LOX-2) decreased lipid accumulation and inflammation, hallmarks of atherosclerosis (8).

Our 15-LOX-2 structure revealed a hydrophobic hairpin structure that projects from the amino-terminal membrane binding domain. Sequence alignment indicates that this hairpin is unique to the 15-LOX-2 isoform and could help position the enzyme at the phospholipid bilayer. Unlike 5-LOX, 15-LOX-2 can process PC-esterified AA (31). This activity suggests a model in which translocation to the membrane increases accessibility to substrate and a tethered enzyme could function at the membrane interface for utilization of AA-bearing phospholipids without the need for a lipase, or for re-esterfication of the product for the oxidized product to enter the PL trafficking pathways.

Structures for two mammalian homologues of $15-\mathrm{LOX}-1$ ( $>80 \%$ identity to human 15 -LOX-1, but $<40 \%$ to 15 -LOX-2) have been reported: one is in the absence and presence of inhibitor (20), and the other is the catalytic domain only with inhibitor (21). These models serve as reliable templates for human 15-LOX-1. The structures of the two 15-LOX isoforms are essentially the same in terms of overall fold: 15 -LOX-2 superimposes with the porcine homologue with an rmsd of $1.62 \AA$ (552 $\mathrm{C} \alpha$ ) and with the uninhibited rabbit 15-LOX-1 with an rmsd of $1.9 \AA(667 \mathrm{C} \alpha)$. However, 15-LOX-1 lacks the extended loop at amino acids 73-85 of 15-LOX-2.

The 15-LOX-2 Inhibitor Defines the Substrate-bound Conformation-Recall that kinetic data indicate that C8E4 is a competitive inhibitor. In addition, AA can adopt an equivalent U-shaped conformation to that of the bound detergent. Moreover, placement of AA tail-first in the C8E4 electron density positions the pentadiene centered at $\mathrm{C} 13$ for attack (Fig. 3, D and $E$ ). This positioning would generate the $15-S$ product, consistent with the product stereochemistry observed for both 15-LOX isoforms. Furthermore, a channel that would allow the 


\section{The Structure of 15-Lipoxygenase-2}

approach of $\mathrm{O}_{2}$ to the activated pentadiene (30) is clearly visible in the surface rendering in Fig. 4. The competitive inhibitor included in the porcine structure indicates a similar, but slightly deeper, cavity to allow three more carbons past that catalytic iron and attack at $\mathrm{C} 10$ (the porcine homologue generates 12-HPETE and attacks at C10). In contrast, the rabbit 15-LOX structure with inhibitor displays a striking conformational change with $\sim 10 \AA$ displacement of helix $\alpha-2$ that likely is unique to the inhibitor included in the crystallization, a conformationally restrained compound that makes contacts inconsistent with the native substrate. Consequently, the 15-LOX-1: inhibitor structure provides solid evidence for malleability of these active sites, and binding site plasticity expands opportunities for isoform-specific inhibitor development.

15-LOX-2 and 5-LOX Differ in the Orientations of Their Common Substrate-The two structures of human LOX now available, the 15-LOX-2 described here and stable 5-LOX (19), can help to formulate novel strategies toward the development of isoform-specific inhibitors because the enzymes display distinctly different active site cavities. A major difference between the two is that the 5-LOX cavity is sealed off by the atypical placement of $\alpha 2$, whereas that in 15-LOX-2 is open to solvent. The closest homologue to 15-LOX-2 in humans is 5-LOX. Recall that if AA were positioned head-first in the 15-LOX-2 active site, it would be transformed to 5-S-HPETE, the 5-LOX product. Extensive biochemical studies have led to a model in which both cavity depth and head versus tail entry of AA combine to explain the varying regio- and stereo-isomer specificities of LOX family members (28). This model predicts that AA enters 5-LOX head-first. We asked whether the head-first entry of AA in 15-LOX-2 as illustrated in Fig. 4C (bottom) might provide insight into how AA binds in 5-LOX. The images and schematic in Fig. 5 reveal that although the internal portions of the LOX cavities are similar in volume, they have dissimilar chemistries. Note that with the loss of a Glu, and the gain of $2 \mathrm{His}$, the inner portion of the 5-LOX cavity is more positive in overall change. The amino acids that hold the catalytic iron in place and position the pentadiene for attack are common to both active sites and composed of invariant or highly conserved amino acids. It is not possible to compare the cavities at the carboxyl end of the 15-LOX-2 active site because this end is tightly corked by $\mathrm{Phe}^{177}$ and $\mathrm{Tyr}^{181}$ in 5-LOX. Although it is tempting to speculate that this end of the site must uncork for AA to enter the 5 -LOX active site, these experiments are beyond the scope of this work.

The Murine Homologue of 15-LOX-2 Is an 8-LOX-15-SHETE (generated by non-enzymatic reduction of 15 -S-HPETE) has been shown to be the LOX product that contributes to atherosclerosis in a murine model system (9). However, silencing expression of murine ALOX15B (which produces 8-HPETE from AA rather than 15-HPETE) mitigated plaque formation (8). These studies appear to be at odds: the former indicates that it is the product of a 15-LOX that promotes plaque formation, whereas one might infer from the latter studies that 8-HETE should be the proinflammatory lipid mediator in the mouse model. This apparent contradiction can be reconciled. Again, the model that proposes that LOX enzymes can differ in terms of whether AA enters head- or tail-first (28) is invoked here.
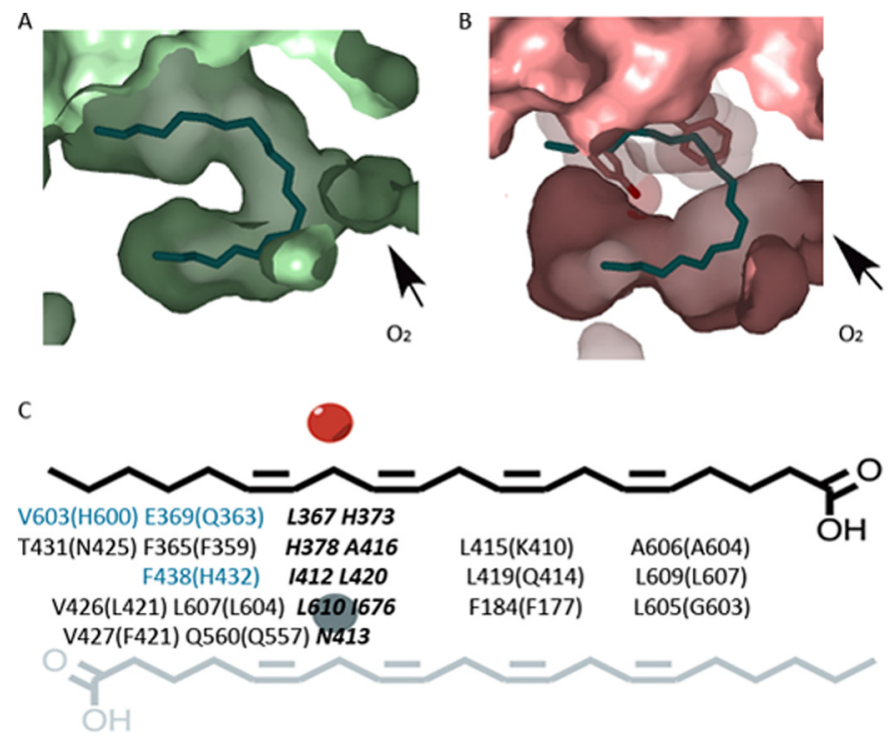

FIGURE 5. The substrate binding cavities of 15-LOX-2 and stable 5-LOX. $A$, a surface rendering of the inhibitor binding site in 15-LOX-2 reveals a cavity that can accommodate AA. The catalytic iron is behind the inhibitor (orientation similar to that in Figs. $3 A$ and $4 A$ ), and an apparent $\mathrm{O}_{2}$ channel is on the upper face (29). B, a similar rendering of the cavity in 5-LOX with the 15-LOX-2 inhibitor superimposed. Note that the volumes of the cavities beyond the $\mathrm{O}_{2}$ channel are similar, but that 5-LOX is "corked" above the channel by two amino acids from $\alpha 2$ (Phe ${ }^{177}, \mathrm{Tyr}^{181}$ ). C, schematic of inverse orientations of AA (black, tail first). Amino acids that line the cavity in 15-LOX-2 are listed with 5-LOX counterparts in parentheses. Invariant amino acids are in boldface type. Amino acid changes that result in an increase in positive change deep in the cavity of 5-LOX are highlighted in blue.

Jisaka et al. (36) demonstrated that the difference in products of 15-LOX-2 and its murine counterpart 8-LOX (87\% sequence identity) can be explained by the substitution of $\mathrm{Asp}^{602} / \mathrm{Val}^{603}$ with $\mathrm{Tyr} / \mathrm{His}$ at the equivalent positions in the $8 S$-enzyme. $\mathrm{Val}^{603}$ is deep in the 15-LOX-2 cavity (Fig. $3 B$ ) where the hydrocarbon end of the substrate sits, and mutation of the murine enzyme to the human sequence (a His to $\mathrm{Val}$ ) at this position transformed the 8-LOX into a 15-LOX by converting an enzyme in which AA enters head-first (as in 5-LOX) to one that enters tail first (as in 15-LOX-2).

Recall that 15-LOX-2 can metabolize PC-esterified AA. It has been reported that murine 8-LOX can also transform phospholipid-esterified AA (31). However, the presence of the phospholipid head group makes head-first entry impossible in the 8-LOX active site, so the murine enzyme cannot produce the $8 S$ isomer from PC-AA. Instead, with PC-AA as the substrate, the murine 8S-LOX is forced to accept tail-first entry. Thus with PC-esterified substrate 8-LOX could be a de facto 15-LOX. Given that 15-LOX-2 displays $\mathrm{Ca}^{2+}$-dependent membrane binding, and the mouse homologue includes those amino acids that confer binding and the putative membrane insertion loop, one might predict that in vivo, the murine enzyme can generate PC-esterified 15-HPETE that ultimately enters the cellular pool of free 15-HETE. It is noteworthy that murine 8-LOX is an anomaly because the other ALOX15B homologues generate 15-HPETE.

Concluding Remarks-In summary, we report here the structure of human 15-LOX-2. As expected 5-LOX and 15-LOX-2 share a common framework, a common catalytic core, and a common substrate. However, the enzymes generate distinct isomers of the product. A comparison of the structures of active site cavities 


\section{The Structure of 15-Lipoxygenase-2}

of this pair of enzymes, along with an understanding of catalytic mechanism, provides insights into substrate recognition.

Acknowledgments-We thank Alan Brash for helpful discussions throughout the course of this work. X-ray data were collected at the Center for Advanced Microstructures and Devices (Baton Rouge), funded in part by the Louisiana Governors' Biotechnology Initiative, and at Beam Line 24-ID-E of Northeastern Collaborative Access Team's at the Advanced Photon Source, supported by award P41 GM103403 from the NIGMS at the National Institutes of Health. Use of the Advanced Photon Source, an Office of Science User Facility operated for the U.S. Department of Energy (DOE) Office of Science by Argonne National Laboratory, was supported by the U.S. DOE under Contract No. DE-ACO206CH11357.

\section{REFERENCES}

1. Harats, D., Shaish, A., George, J., Mulkins, M., Kurihara, H., Levkovitz, H., and Sigal, E. (2000) Overexpression of 15-lipoxygenase in vascular endothelium accelerates early atherosclerosis in LDL receptor-deficient mice. Arterioscler. Thromb. Vasc. Biol. 20, 2100-2105

2. Sendobry, S. M., Cornicelli, J. A., Welch, K., Bocan, T., Tait, B., Trivedi, B. K., Colbry, N., Dyer, R. D., Feinmark, S. J., and Daugherty, A. (1997) Attenuation of diet-induced atherosclerosis in rabbits with a highly selective 15-lipoxygenase inhibitor lacking significant antioxidant properties. Br. J. Pharmacol. 120, 1199-1206

3. Bocan, T. M., Rosebury, W. S., Mueller, S. B., Kuchera, S., Welch, K., Daugherty, A., and Cornicelli, J. A. (1998) A specific 15-lipoxygenase inhibitor limits the progression and monocyte-macrophage enrichment of hypercholesterolemia-induced atherosclerosis in the rabbit. Atherosclerosis 136, 203-216

4. Funk, C. D., Chen, X. S., Johnson, E. N., and Zhao, L. (2002) Lipoxygenase genes and their targeted disruption. Prostaglandins Other Lipid. Mediat. 68, 303-312

5. Funk, C. D. (2006) Lipoxygenase pathways as mediators of early inflammatory events in atherosclerosis. Arterioscler. Thromb. Vasc. Biol. 26, $1204-1206$

6. Gertow, K., Nobili, E., Folkersen, L., Newman, J. W., Pedersen, T. L., Ekstrand, J., Swedenborg, J., Kühn, H., Wheelock, C. E., Hansson, G. K., Hedin, U., Haeggström, J. Z., and Gabrielsen, A. (2011) 12- and 15-lipoxygenases in human carotid atherosclerotic lesions: associations with cerebrovascular symptoms. Atherosclerosis 215, 411-416

7. Wuest, S. J., Crucet, M., Gemperle, C., Loretz, C., and Hersberger, M. (2012) Expression and regulation of 12/15-lipoxygenases in human primary macrophages. Atherosclerosis 225, 121-127

8. Magnusson, L. U., Lundqvist, A., Karlsson, M. N., Skålén, K., Levin, M., Wiklund, O., Borén, J., and Hultén, L. M. (2012) Arachidonate 15-lipoxygenase type B knockdown leads to reduced lipid accumulation and inflammation in atherosclerosis. PLoS One 7, e43142

9. Kotla, S., Singh, N. K., Heckle, M. R., Tigyi, G. J., and Rao, G. N. (2013) The transcription factor CREB enhances interleukin-17A production and inflammation in a mouse model of atherosclerosis. Sci. Signal. 6, ra83

10. Brash, A. R. (1999) Lipoxygenases: occurrence, functions, catalysis, and acquisition of substrate. J. Biol. Chem. 274, 23679-23682

11. Bazan, N. G., Musto, A. E., and Knott, E. J. (2011) Endogenous signaling by omega-3 docosahexaenoic acid-derived mediators sustains homeostatic synaptic and circuitry integrity. Mol. Neurobiol. 44, 216-222

12. Boyington, J. C., Gaffney, B. J., and Amzel, L. M. (1993) The three-dimensional structure of an arachidonic acid 15-lipoxygenase. Science 260, $1482-1486$

13. Otwinowski, Z., and Minor, W. (1997) Processing of x-ray diffraction data collected in oscillation mode in Methods Enzymology (Carter, C. W., and Sweet, R. M., eds) pp. 307-326, Academic Press, New York

14. Zwart, P. H., Afonine, P. V., Grosse-Kunstleve, R. W., Hung, L. W., Ioerger, T. R., McCoy, A. J., McKee, E., Moriarty, N. W., Read, R. J., Sacchettini, J. C., Sauter, N. K., Storoni, L. C., Terwilliger, T. C., and Adams, P. D.
(2008) Automated structure solution with the PHENIX suite. Methods Mol. Biol. 426, 419-435

15. Emsley, P., and Cowtan, K. (2004) Coot: model-building tools for molecular graphics. Acta Crystallogr. D Biol. Crystallogr. 60, 2126-2132

16. Cleland, W. W. (1979) Statistical analysis of enzyme kinetic data. Methods Enzymol. 63, 103-138

17. Boldog, T., Li, M., and Hazelbauer, G. L. (2007) Using Nanodiscs to create water-soluble transmembrane chemoreceptors inserted in lipid bilayers. Methods Enzymol. 423, 317-335

18. Denisov, I. G., Grinkova, Y. V., Lazarides, A. A., and Sligar, S. G. (2004) Directed self-assembly of monodisperse phospholipid bilayer Nanodiscs with controlled size. J. Am. Chem. Soc. 126, 3477-3487

19. Gilbert, N. C., Bartlett, S. G., Waight, M. T., Neau, D. B., Boeglin, W. E., Brash, A. R., and Newcomer, M. E. (2011) The structure of human 5-lipoxygenase. Science 331, 217-219

20. Choi, J., Chon, J. K., Kim, S., and Shin, W. (2008) Conformational flexibility in mammalian 15S-lipoxygenase: Reinterpretation of the crystallographic data. Proteins 70, 1023-1032

21. Xu, S., Mueser, T. C., Marnett, L. J., and Funk, M. O., Jr. (2012) Crystal structure of 12-lipoxygenase catalytic-domain-inhibitor complex identifies a substrate-binding channel for catalysis. Structure 20, 1490-14.97

22. Eek, P., Järving, R., Järving, I., Gilbert, N. C., Newcomer, M. E., and Samel, N. (2012) Structure of a calcium-dependent 11R-lipoxygenase suggests a mechanism for $\mathrm{Ca}^{2+}$ regulation. J. Biol. Chem. 287, 22377-22386

23. Neau, D. B., Gilbert, N. C., Bartlett, S. G., Boeglin, W., Brash, A. R., and Newcomer, M. E. (2009) The 1.85 A structure of an 8R-lipoxygenase suggests a general model for lipoxygenase product specificity. Biochemistry 48, 7906-7915

24. Boyington, J. C., Gaffney, B. J., and Amzel, L. M. (1993) The three-dimensional structure of an arachidonic acid 15-lipoxygenase. Science 260, $1482-1486$

25. Hammarberg, T., Provost, P., Persson, B., and Rådmark, O. (2000) The $\mathrm{N}$-terminal domain of 5-lipoxygenase binds calcium and mediates calcium stimulation of enzyme activity. J. Biol. Chem. 275, 38787-38793

26. Kulkarni, S., Das, S., Funk, C. D., Murray, D., and Cho, W. (2002) Molecular basis of the specific subcellular localization of the C2-like domain of 5-lipoxygenase. J. Biol. Chem. 277, 13167-13174

27. Kilty, I., Logan, A., and Vickers, P. J. (1999) Differential characteristics of human 15-lipoxygenase isozymes and a novel splice variant of 15S-lipoxygenase. Eur. J. Biochem. 266, 83-93

28. Schneider, C., Pratt, D. A., Porter, N. A., and Brash, A. R. (2007) Control of oxygenation in lipoxygenase and cyclooxygenase catalysis. Chem. Biol. 14, $473-488$

29. Murphy, R. C., and Gijón, M. A. (2007) Biosynthesis and metabolism of leukotrienes. Biochem. J. 405, 379-395

30. Knapp, M. J., Seebeck, F. P., and Klinman, J. P. (2001) Steric control of oxygenation regiochemistry in soybean lipoxygenase-1. J. Am. Chem. Soc. 123, 2931-2932

31. Coffa, G., and Brash, A. R. (2004) A single active site residue directs oxygenation stereospecificity in lipoxygenases: Stereocontrol is linked to the position of oxygenation. Proc. Natl. Acad. Sci. U.S.A.

32. Hutchins, P. M., and Murphy, R. C. (2012) Cholesteryl ester acyl oxidation and remodeling in murine macrophages: formation of oxidized phosphatidylcholine. J. Lipid Res. 53, 1588-1597

33. Brash, A. R., Boeglin, W. E., and Chang, M. S. (1997) Discovery of a second 15S-lipoxygenase in humans. Proc. Natl. Acad. Sci. U.S.A. 94, 6148-6152

34. Danielsson, K. N., Rydberg, E. K., Ingelsten, M., Akyürek, L. M., Jirholt, P., Ullström, C., Forsberg, G. B., Borén, J., Wiklund, O., and Hultén, L. M. (2008) 15-Lipoxygenase-2 expression in human macrophages induces chemokine secretion and T cell migration. Atherosclerosis 199, 34-40

35. Hultén, L. M., Olson, F. J., Aberg, H., Carlsson, J., Karlström, L., Borén, J., Fagerberg, B., and Wiklund, O. (2010) 15-Lipoxygenase-2 is expressed in macrophages in human carotid plaques and regulated by hypoxia-inducible factor-1 $\alpha$. Eur. J. Clin. Invest. 40, 11-17

36. Jisaka, M., Kim, R. B., Boeglin, W. E., and Brash, A. R. (2000) Identification of amino acid determinants of the positional specificity of mouse 8S-lipoxygenase and human 15S-lipoxygenase-2. J. Biol. Chem. 275, 1287-1293 